\title{
Handling Test of Eye Drop Dispenser- Comparison of Unit-Dose Pipettes with Conventional Eye Drop Bottles
}

\author{
Minna Parkkari, ${ }^{1}$ Terho Latvala, ${ }^{2,3}$ and Auli Ropo ${ }^{2}$
}

\begin{abstract}
Purpose: The aims of this study were to investigate how elderly people handle single-use eye drop dispensers (unit-dose pipettes) and to compare the performance with conventional eye drop bottles.

Methods: In this open-label study, the handling of unit-dose pipettes and conventional eye drop bottles was compared in 41 elderly people who had little or no prior regular use of eye drop dispensers. The participants tested both types of dispenser once, and the following 7 variables were studied: ease/difficulty of opening the dispenser; influence of the size for handling of the dispenser; influence of the shape for handling of the dispenser; observation of the contents in the dispenser; the feeling of the dispenser in the hand; ease/difficulty of drop instillation on the eye from the dispenser; and overall performance of the eye drop dispenser. The dispensers contained isotonic saline, and a visual analog scale was used for assessment of each of the above variables.

Results: The mean age of the participants was 73 years. A statistically significant difference in favor of the unitdose pipettes was found with respect to observation of the contents in the dispenser, ease of administration, and the overall performance. Women regarded the unit-dose pipettes generally better than the bottles, but such a difference was not seen in men.
\end{abstract}

Conclusions: The study participants managed the unit-dose pipettes at least as well as the conventional eye drop bottles. If anything, the unit-dose pipettes appeared to be easier to use.

\section{Introduction}

$\mathbf{M}$ ANY PRESERVATIVES USED in eye drops have toxic effects. Benzalkonium chloride (BAC) is the most commonly used preservative. High concentrations of BAC may cause inflammatory changes and apoptosis in the conjunctival and corneal epithelium during chronic use. ${ }^{1-5}$ Despite being a quaternary ammonium compound with poor penetration into the eye, it has been suggested that BAC increases the risk for cystoid macular edema in conjunction with cataract surgery, 6,7 and that chronic use of eye drops containing BAC reduces the success rate of trabeculectomy surgery because of subepithelial fibrosis and inflammatory changes in the conjunctiva. ${ }^{8-10}$ Evidence from in vitro studies suggest that BAC has negative effects on the corneal endothelium, ${ }^{11}$ trabecular meshwork cells, ${ }^{12}$ and lens epithelium. ${ }^{13}$ It is obvious that eye drops without BAC would be preferred as they are less damaging to the eye.

In single-use eye drop dispensers, for example, unit-dose pipettes, no preservative is necessary, and therefore, all the side effects caused by BAC can be avoided. However, in previous studies it has been suspected that elderly patients may have difficulties in managing single-use dispensers, and the instillation of the eye drops may fail more often compared with conventional eye drop bottles. ${ }^{14,15}$ Obviously, this may be due to several factors such as the size and shape of the dispensers and softness of the plastic material. The purpose of this study was to investigate how elderly participants manage unit-dose pipettes in comparison to conventional eye drop bottles.

\section{Methods}

This study was performed in accordance with the tenets of the Declaration of Helsinki and the study protocol was submitted to the Independent Ethics Committee of Tampere University Hospital for approval. Written informed consent was obtained from all participants prior to inclusion. The study adopted an open-label protocol. A total of 41 healthy participants who attended 4 private ophthalmology clinics in Tampere, Finland, were included. The inclusion criteria

\footnotetext{
${ }^{1}$ Department of Ophthalmology, University of Tampere, Tampere, Finland.

${ }^{2}$ Santen Oy, Helsinki, Finland.

${ }^{3}$ Helsinki University Hospital, Eye Clinic, Helsinki, Finland.
} 
comprised a minimum age of 60 years and no regular prior use of eye drops or contact lenses. Sporadic previous use of eye drops, such as that in conjunction with ocular inflammation, was allowed. Participants were excluded if they were suffering from ophthalmic diseases such as acute or chronic inflammation, infection or any other ophthalmic disease treated regularly with eye drop dispensers, had poor vision including complicated refractive error, or had systemic diseases that would negatively affect their ability to instill eye drops, such as rheumatoid arthritis, arthrosis in the fingers, and cervical spondylarthritis, as well as neurological and psychiatric disorders.

The single-use eye drop dispensers (unit-dose pipettes) and the conventional eye drop bottles were manufactured using transparent low-density polyethylene plastic. The screw cap of the bottles was manufactured using white highdensity polyethylene plastic. Both types of dispensers (Fig. 1) were filled with a sterile isotonic saline solution $(0.3 \mathrm{~mL}$ in the unit-dose pipettes and $5 \mathrm{~mL}$ in the eye drop bottles).

An eye drop bottle and a single-dose pipette were given to the study participants at the same time. They were given standard written instructions on how to use the dispensers and were not assisted in any way to interpret the instructions, or instill the eye drop. The study participants tested the eye drop dispensers and instilled a drop into the conjunctival sac/corneal surface from the unit-dose pipette and the eye drop bottle. The eye drop was always instilled into the same eye. The study participants were free to choose either their dominant or nondominant hand, but they had to use the same hand with both types of dispensers. The different variables were assessed on a $10 \mathrm{~cm}$ visual analog scale (VAS) by the study participants. The left end of VAS corresponded to "good/easy," whereas the right end corresponded to "poor/difficult." The participants were asked to assess the variables by marking the scale with the distance to the mark from the left end of the line measured in millimeters. The following variables were studied:

1. Ease/difficulty to open the dispenser;

2. Impact of size on the handling of the dispenser;

3. Impact of shape on the handling of the dispenser;

4. Observation of the content (volume of fluid) in the dispenser;

5. Feeling of the dispenser in the hand;

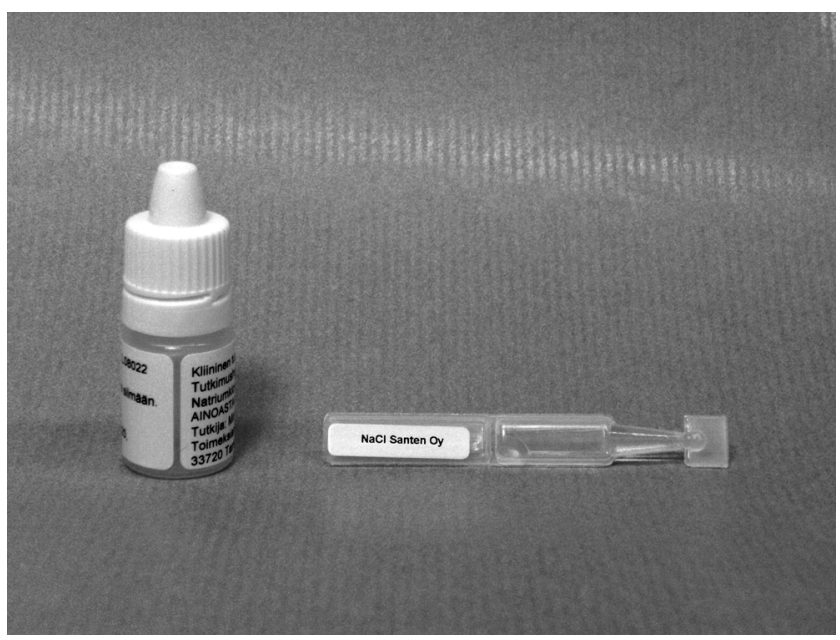

FIG. 1. Types of dispensers used in the study.
6. Ease/difficulty of drop instillation on the eye from the dispenser;

7. Overall impression of the handling/performance of the dispenser.

The mean differences between the 2 study groups for each of these variables were calculated with $95 \%$ confidence interval. The results were statistically analyzed using a paired $t$-test or, if the assumption of "normal distribution" did not apply, a permutation test or Wilcoxon's test was used. The internal consistency of the variables between test groups was checked using Cronbach's alpha. The results were tabulated and graphically displayed using a modification of the Bland and Altman presentation, or Lin's concordance.

\section{Results}

A total of 41 participants were included in the study; 1 participant failed to open the screw cap of the eye drop bottle and, therefore, could not continue the test. All the other participants were able to test both types of eye drop dispensers and completed the study successfully. The demographic characteristics of the study population are presented in Table 1. Of the participants, 11 (all women) had previously occasionally used eye drops. Of the previous eye drop medications, 11 were instilled from bottles and 4 from unitdose dispensers.

The results of the 7 variables based on all participants are shown in Fig. 2. The study participants reported that it was significantly easier to visualize the content in the unit-dose pipettes compared with the bottles $(P<0.001)$. Importantly, the instillation of the eye drops was easier using the unitdose pipettes $(P=0.0042)$ and the overall performance of the pipettes was better than that of conventional eye drop bottles $(P<0.001)$. There was no significant difference between the results from participants aged younger than 70 years compared with those aged older than 70 years.

For the participants who had previous occasional use of eye drops, the unit-dose pipettes were easier to open, but for those with no previous experience of eye drops no difference was seen between the unit-dose pipettes and the eye drop bottles (Fig. 3); the difference between the groups was significant $(P=0.0045)$. The observation of the content was significantly better in the unit-dose pipettes compared with the eye drop bottles for both categories of participants. Instillation of the eye drop was judged to be easier among those using the unit-dose pipettes compared with the eye drop bottles in participants without previous experience of eye drops. The overall performance appeared to be better for

Table 1. Demographic Characteristics of Study Population

\begin{tabular}{lccc}
\hline Characteristic & $\begin{array}{c}\text { Men } \\
(\mathrm{n}=12)\end{array}$ & $\begin{array}{c}\text { Women } \\
(\mathrm{n}=29)\end{array}$ & $\begin{array}{c}\text { All } \\
(\mathrm{N}=41)\end{array}$ \\
\hline Age, years & & & \\
Mean \pm SD & $73.1 \pm 7.1$ & $72.9 \pm 6.9$ & $73.0 \pm 6.9$ \\
Minimum-maximum & $60-83$ & $60-84$ & $60-84$ \\
$\begin{array}{l}\text { No previous experience } \\
\text { of eye drops, } n\end{array}$ & 12 & 18 & 30 \\
\hline
\end{tabular}

Abbreviation: SD, standard deviation. 


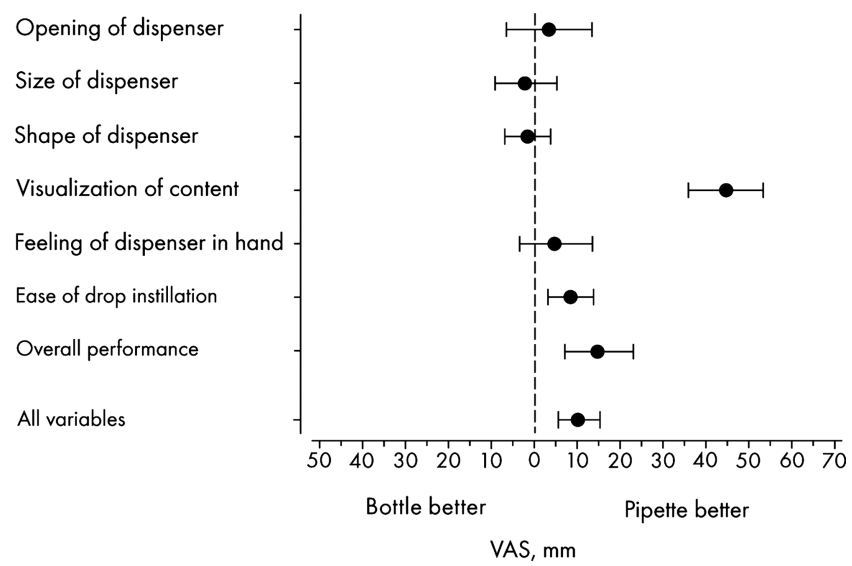

FIG. 2. Differences in the results between unit-dose pipettes and conventional eye drop bottles. Mean value with $95 \%$ confidence interval $(n=41)$ according to the VAS. VAS, visual analog scale.

the unit-dose pipettes in both categories of participants compared with the eye drop bottles.

Men reported that the eye drop bottles were easier to open compared with the unit-dose pipettes, whereas the opposite was found for women (Fig. 4); the difference between the groups was statistically significant $(P=0.0025)$. Both men and women reported that the observation of the contents was much easier in the unit-dose pipettes than in the eye drop bottles. Except for the opening of the dispensers, there was little difference between men and women in the variables studied.

\section{Discussion}

The results of this study show that the differences in the handling/performance of unit-dose pipettes compared with conventional eye drop bottles were generally relatively small. However, the mean difference, based on all study variables, was usually statistically significantly in favor of

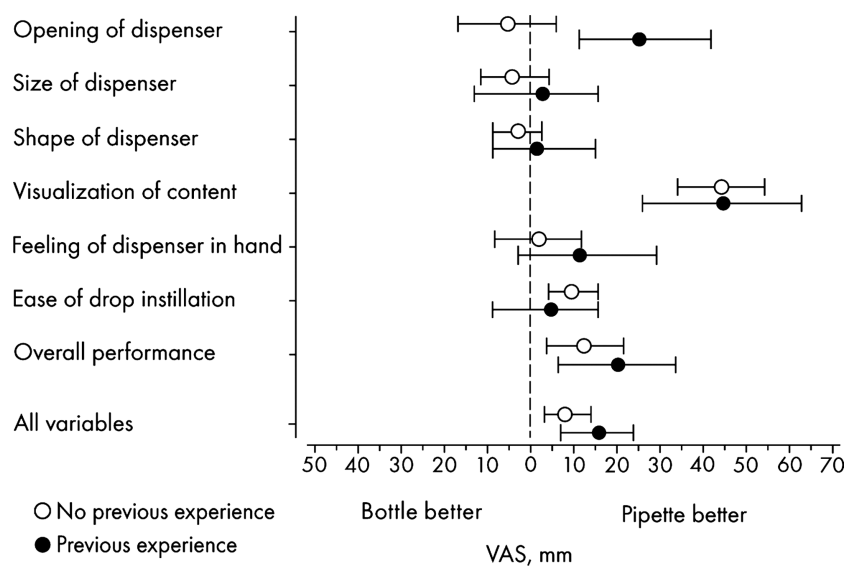

FIG. 3. Differences in the results between unit-dose pipettes and conventional eye drop bottles in participants without and with previous experience of eye drop instillation. Mean value with $95 \%$ confidence interval (number of participants without previous experience $=30$; number of participants with previous experience $=11$ ). VAS, visual analog scale.

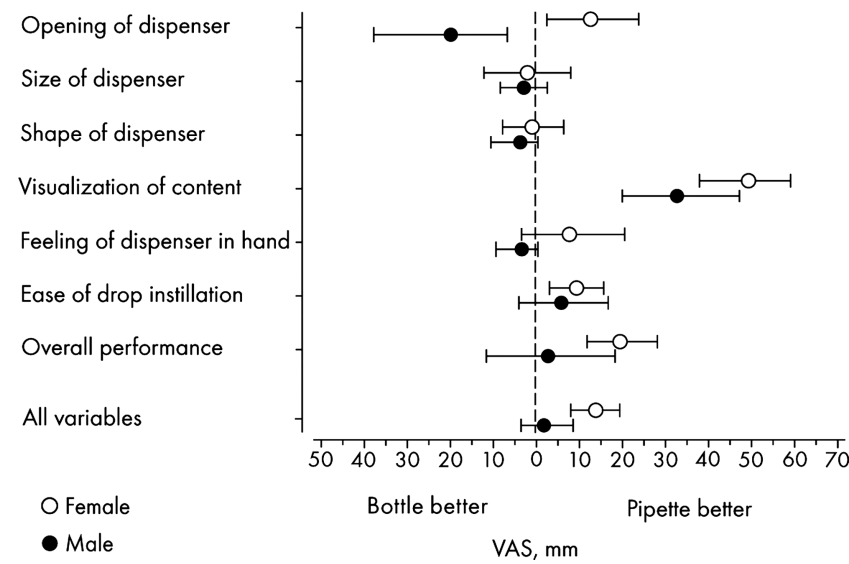

FIG. 4. Differences in the results between unit-dose pipettes and conventional eye drop bottles in men and women. Mean value with $95 \%$ confidence interval (number of women $=30$; number of men $=11$ ). VAS, visual analog scale.

the unit-dose pipettes. The unit-dose pipettes appeared to be preferred by women because opening of the pipette as well as the instillation of the eye drop was easier compared with the eye drop bottles. The fact that some women had sporadic, previous experience of unit-dose dispensers may have been a contributing factor to this finding. The preference for unit-dose pipettes by women could be as a result of their handling of make-up on eyelids and eyelashes. Both women and men could observe the contents in the unit-dose pipettes significantly better than that in eye drop bottles; however, men regarded eye drop bottles easier to open.

In a recent study by Dietlein et al., ${ }^{15}$ it was reported that glaucoma or dry eye patients, aged 80 years or older, experienced more difficulty in opening and applying drops from single-dose containers compared with conventional eye drop bottles. Decreased visual acuity as well as lack of experience was significantly correlated with difficulties in the correct instillation of eye drops. Contributing factors to the discrepancy between the results of the previous study and this study may comprise physical differences in the single-dose dispensers and perhaps the older age of the participants involved (mean age, 84 years vs. 73 years). In the study by Dietlein et al., participants had used eye drop bottles earlier, but only a fraction had previous experience with single-use eye-drop containers $(27 \%-39 \%){ }^{15}$ The problems with selfinstillation from the single-dose containers were associated with lack of experience with using them. The authors concluded that there was a training effect involved. This may have favored the multidose bottles. One notable difference between this study and the study by Dietlein et al. was that in this study written instructions were provided to the participants. ${ }^{15}$

The participants of this study, who had a mean age of 73 years, had good visual acuity and no systemic diseases that could negatively affect the instillation of the eye drops. Therefore, although in a relatively healthy elderly population the unit-dose pipettes appeared to be somewhat better than the conventional eye drop bottles, it is difficult to predict if this is true in handicapped patients, for example, those who suffer from rheumatoid arthritis or reduced visual acuity. A similar handling test of eye drop dispensers in handicapped patients may be necessary for addressing this question. 
In conclusion, polyethylene unit-dose pipettes were at least as easy to manage as conventional eye drop bottles and, if anything, appeared to be better in the population studied. Thus, presentation of preservative-free medications in unitdose pipettes, of the kind investigated in this study, would appear to be adequate for self-instillation of the medication by patients.

\section{Acknowledgments}

The authors received editorial and writing support in the preparation of this manuscript. This study was funded by Santen Oy, Tampere, Finland. The authors thank Hannu Kautiainen for his statistical contributions to this study.

\section{Author Disclosure Statement}

M. Parkkari has received funding for research carried out in this work from Santen Oy, Finland. No competing financial interests exist. T. Latvala and A. Ropo are employees of Santen Oy. The authors have no proprietary interest in the study.

\section{References}

1. Pisella, P.J., Deppasch, C., Hamard, P., et al. Conjunctival proinflammatory and proapoptotic effects of latanoprost and preserved and unpreserved timolol: an ex vivo and in vitro study. Invest. Ophthalmol. Vis Sci. 45:1360-1368, 2004.

2. Baudouin, C., Riancho, L., Warnet, J.M., and Brignol, F. In vitro studies of antiglaucomatous prostaglandin analogues: travoprost with and without benzalkonium chloride and preserved latanoprost. Invest. Ophthalmol. Vis. Sci. 48:4123-4128, 2007.

3. Whitson, J.T., Cavanagh, H.D., Lakhsman, N., and Petroll, W.M. Assessment of corneal epithelial integrity after acute exposure to ocular hypotensive agents preserved with and without benzalkonium chloride. Adv. Ther. 23:663-671, 2006.

4. Noecker, R.J., Herrygers, L.A., and Anwaruddin, R. Corneal and conjunctival changes caused by commonly used glaucoma medications. Cornea 23:490-496, 2004.

5. Pisella, P.J., Fillacier, K., Elena, P.P., Debbash, C., and Baudouin, C. Comparison of the effects of preserved and unpreserved formulations of timolol on the ocular surface of albino rabbits. Ophthalmic Res. 32:3-8, 2000.

6. Miyake, K., Ota, I., Ibraki, N., et al. Enhanced disruption of the blood-aqueous barrier and the incidence angiographic cystoid macular edema by topical timolol and its preserva- tive in early postoperative pseudophakia. Arch. Ophthalmol. 119:387-394, 2001.

7. Miyake, K., Ibariki, N., Goto, Y., et al. ESCRS Binkhorst lecture 2002: pseudophakic preservative maculopathy. J. Cataract Refract. Surg. 29:1800-1810, 2004.

8. Broadway, D.C., Grierson, I., O'Brien, C., and Hitchings, R.A. Adverse effects of topical antiglaucoma medication. I. The conjunctival cell profile. Arch. Ophthalmol. 112:14371445, 1994.

9. Broadway, D.C., Grierson, I., O'Brien, C., and Hitchings, R.A. Adverse effect of topical antiglaucoma medication. II. The outcome of filtration surgery. Arch. Ophthalmol. 112: 1446-1454, 1994.

10. Baudouin, C. Mechanisms of failure of glaucoma filtering surgery: a consequence of antiglaucomatous drugs. Int. J. Clin. Pharmacol. Res. 16:29-41, 1996.

11. Liu, H., Routley, I., and Teichmann, K.D. Toxic endothelial cell destruction from intraocular benzalkonium chloride. J. Cataract Refract. Surg. 27:1746-1750, 2001.

12. Hamard, P., Blondin, C., Debbash, C., Warnet, J.M., Baudouin, C., and Brignole, F. In vitro effects of preserved and unpreserved antiglaucoma drugs on apoptotic marker expression by human trabecular cells. Graefes Arch. Clin. Exp. Ophthalmol. 241:1037-1043, 2003.

13. Goto, Y., Ibraki, N., and Miyake, K. Human lens epithelial cell damage and stimulation of their secretion of chemical mediators by benzalkonium chloride rather than latanoprost and timolol. Arch. Ophthalmol. 121:835-839, 2003.

14. Oldham, G.B., and Andrews, V. Control of microbial contamination in unpreserved eye drops. Br. J. Ophthalmol. 80:588-591, 1996.

15. Dietlein, T.S., Jordan, J.F., Luke, C., et al. Self-application of single-use eyedrop containers in an elderly population: comparisons with standard eyedrop bottle and with younger patients. Acta Ophthalmol. 86:856-859, 2008.

Received: October 14, 2009 Accepted: April 16, 2010

Address correspondence to: Dr. Minna Parkkari

Department of Ophthalmology University of Tampere Simolankatu 1

33270 Tampere Finland

E-mail: minna.parkkari@uta.fi 